\title{
A MULTIAXIAL CRITERION FOR NOTCH FATIGUE USING A CRITICAL-DISTANCE METHOD
}

\author{
Andrea Carpinteri, Andrea Spagnoli, Sabrina Vantadori, Danilo Viappiani \\ Department of Civil and Environmental Engineering \& Architecture \\ University of Parma \\ Italy
}

\begin{abstract}
In the present paper, a high-cycle critical plane-based multaxial fatigue criterion, recently proposed by the authors to determine the fatigue strength of smooth components, is extended to notched components by using the so-called point method. Accordingly, once the location of the 'hot spot' on the notch surface is determined, the orientation of the critical plane (material plane where fatigue strength assessment has to be performed) is assumed to be correlated with the principal stress directions in the hot spot itself. Some experimental results related to round bars with a surface circular notch (an artificially drilled surface hole) submitted to cyclic loading are compared with the theoretical predictions of the proposed extension. The comparison, which is instrumental in highlighting the notch size-effect (as the hole diameter varies) under uniaxial and biaxial far-field stress conditions, appears to be quite satisfactory.
\end{abstract}

\section{Introduction}

Since the fundamental works of Neuber [1] and Peterson [2], notch fatigue has attracted a great deal of attention in the research community. Among others and in line with such works, some recent approaches to notch fatigue are based on the idea of considering a certain volume in the vicinity of the notch surface to assess the fatigue strength of the component. In this way, the influence of the stress/strain gradient due to the notch on the fatigue strength can be taken into account. It is worth mentioning, for instance, the critical layer approach of Flavenot \& Skalli [3], the general criterion of Seweryn \& Mroz [4], as well as the criterion of Papadopoulos [5] which, conversely to the previous proposals, explicitly accounts for stress gradient instead of considering a finite volume around the notch tip.

Another paper to be mentioned is Ref. [6] by Tanaka. In this line, a recent proposal to estimate the fatigue limit of notched components is that by Taylor [7]. Accordingly, the endurance limit condition in a notched component occurs either when the amplitude of a relevant stress component (e.g. the normal stress perpendicular to the notch bisector), computed from a linear elastic analysis at a given distance from the notch tip (point method), is equal to $\sigma_{a f}\left(\sigma_{a f}=\right.$ fatigue limit measured from a smooth specimen), or when the averaged value of such a stress amplitude over a line (line method) or a semi-circular area (area method) ahead of the notch tip is equal to $\sigma_{a f}$. Such so-called critical-distance methods assume that the distance from the notch tip, the length of the line and the radius of the area ahead of the notch tip are equal to $L / 2,2 L$ and $L$, respectively, where $L$ is a material constant equal to the ElHaddad 'intrinsic' crack length [8], that is:

$$
L=\frac{1}{\pi}\left(\frac{\Delta K_{t h}}{\sigma_{a f}}\right)^{2}
$$

$\Delta K_{t h}$ being the threshold range of the stress intensity factor for long cracks.

Taylor has shown that these methods can successfully be applied to predict the endurance limit of components containing either sharp or blunt or short notches [7,9] subjected to uniaxial cyclic loading. The predicting capability of the line method seems to depend on a theoretical argument in the case of sharp notches, which are characterised by non-propagating cracks at the endurance limit [10]. As a matter of fact, the stress intensity factor range of a non-propagating crack of length $2 L$ originating from the tip of the notch has been shown to attain the threshold value $\Delta K_{\text {th }}$ of the material under a stress field which, in the uncracked body, presents an averaged value of the normal stress amplitude along the crack line equal to $\sigma_{a f}[11]$. 
When a notched component is subjected to a far-field multiaxial cyclic loading, appropriate stress components ahead of the notch tip must be selected in order to exploit the above critical-distance methods. As has been shown in the works by Susmel \& Taylor [12] and Naik et al [13], critical plane-based multiaxial criteria can be used in conjunction with one of the criticaldistance methods. In the present paper, we propose an extension of our criterion presented in Refs [14-19] to multiaxial notch fatigue by using the point method.

\section{Extension of the Carpinteri-Spagnoli (C-S) criterion to multiaxial notch fatigue}

According to this criterion, the fatigue limit estimation under multiaxial high-cycle fatigue is performed by analysing the timevarying stress tensor (determined from a linear elastic analysis) at a given point of the material. In the case of stress components assumed to be periodic functions of time, the main steps of the criterion are as follows [14-18]:

- Averaged directions of the principal stresses are determined on the basis of their instantaneous directions (two material parameters are required at this step: the fatigue limit $\sigma_{a f}$ under fully reversed normal stress, and the corresponding slope $m$ of the S-N curve in the uniaxial high-cycle regime);

- The orientation of the verification material plane (hereafter termed 'critical plane', where fatigue strength assessment has to be performed) and that of the final fracture plane are linked to the averaged directions of the principal stress axes (one further material parameter is required at this step: the fatigue limit $\tau_{a f}$ under fully reversed shear stress);

- The maximum value of normal stress and the amplitude of shear stress, both acting on the critical plane, are determined in a loading cycle;

- $\quad$ The fatigue strength estimation is performed through a quadratic combination of normal and shear stress components acting on the critical plane.

As has been pointed out by Brown and Miller [20], fatigue crack propagation can be distinguished into two stages: a first one (Stage 1) in which a crack nucleates (near the external surface of a structural component) along a shear slip plane (Mode II, fatigue crack initiation plane), and a second one (Stage 2) in which crack propagation occurs in a plane normal to the direction of the maximum principal stress (Mode I, final fatigue fracture plane).

By comparing theoretical and experimental results [16], the normal to the estimated final fatigue fracture plane of Stage 2, which is the one observed post mortem at the macro level, has been assessed to be coincident with the averaged direction $\hat{1}$ of the maximum principal stress $\sigma_{1}$. This implies that the orientation of the final fatigue fracture plane depends on the timevarying stress state and the material parameters $\sigma_{a f}$ and $m_{\sigma}=-1 / m$.

The orientation of the critical plane has been proposed to be correlated with the averaged directions of the principal stress axes [16]. In more detail, the empirical expression for $\delta$ (with $\delta=$ angle between the normal $\mathrm{w}$ to the critical plane and the averaged direction $\hat{1}$ of the maximum principal stress $\sigma_{1}$, where w belongs to the principal plane $\hat{1} \hat{3}$ as is shown in Fig. 1) is given by:

$$
\delta=\frac{3 \pi}{8}\left[1-\left(\frac{\tau_{a f}}{\sigma_{a f}}\right)^{2}\right]
$$

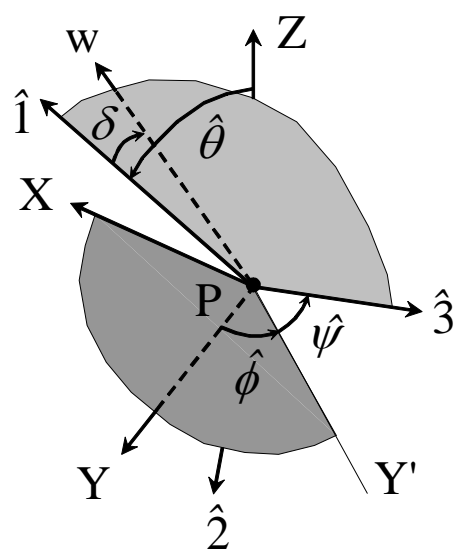

Figure 1. Correlation between averaged principal stress directions $\hat{1} \hat{2} \hat{3}$ and normal $\mathbf{w}$ to the critical plane. 
Equation 2 is valid for hard metals which are characterised by values of the ratio $\tau_{\text {af }} / \sigma_{\text {af }}$ ranging from $1 / \sqrt{3}$ to 1 (note that the lower limit of $\tau_{a f} / \sigma_{a f}$ corresponds to the Von Mises strength criterion of mild metals for static loading). In the light of the above, the orientation of the critical plane depends on the time-varying stress state as well as the material parameters $\sigma_{a f}, \tau_{a f}$ and $m_{\sigma}$.

When the ratio $\tau_{a f} / \sigma_{a f}$ tends to $1 / \sqrt{3}$ (threshold between mild and hard metals), the off angle $\delta$ tends to $\pi / 4$. In this case, the critical plane tends to coincide with the actual fatigue crack initiation plane (Stage 1). This is justified by the fact that, in the high-cycle fatigue regime, the microcrack initiation stage is the critical one for such materials. On the other hand, when the ratio $\tau_{a f} / \sigma_{a f}$ tends to 1 (threshold between hard and extremely hard metals), the off angle $\delta$ tends to zero. This is in line with the fact that such materials have predominantly Stage 2 crack growth and, hence, the critical plane is assumed to be the final fatigue fracture plane. In conclusion, the introduction of the off angle $\delta$ allows us to consider both shear stress (Mode II) and normal stress (Mode I) mechanisms (the governing mechanism depends on the fatigue behaviour of the material and the stress state) of crack initiation pointed out by Socie [21].

For multiaxial constant-amplitude cyclic loading, the normal stress vector $\mathbf{N}$ and the shear stress vector $\mathbf{C}$ lying on the critical plane are periodic functions of time. The direction of the normal stress $\mathbf{N}(\mathrm{t})$ is fixed with respect to time: consequently, the mean value $N_{m}$ and the amplitude $N_{a}$ of the normal stress can readily be calculated. On the other hand, the definitions of the shear stress mean value $C_{m}$ and amplitude $C_{a}$ are not unique, owing to the generally time-varying direction of $\mathbf{C}$. The procedure proposed by Papadopoulos [22] has been adopted in Refs $[17,18]$ to determine $C_{m}$ and $C_{a}$.

The multiaxial fatigue limit condition occurs $[17,18]$ when the equivalent stress amplitude $\sigma_{e q, a}$ (a nonlinear combination of the maximum normal stress, $N_{\max }=N_{m}+N_{a}$, and the shear stress amplitude, $C_{a}$, acting on the critical plane) is equal to $\sigma_{a f}$ :

$$
\sigma_{e q, a}=\sqrt{N_{\max }^{2}+\left(\frac{\sigma_{a f}}{\tau_{a f}}\right)^{2} C_{a}^{2}}=\sigma_{a f}
$$

Such a criterion is to some extent reminiscent of the well-known Gough ellipse [23]. However, differently from the Gough's criterion, the proposed criterion considers stress components acting on the critical plane and, hence, is capable of implicitly accounting for loading non-proportionality (e.g. the critical plane orientation depends on the phase angles between the stress components). Note that some well-established experimental findings are included in Eq. 3, namely: the mean shear stress $C_{m}$ does not affect the high-cycle fatigue strength of hard metals [23], whereas the effect of the mean normal stress $N_{m}$ is taken into account.

Consider a traction-free notch surface contained in a body submitted to a constant-amplitude loading. At any point on the notch surface a principal stress is always null and its direction is normal to the notch surface. The point $\mathrm{H}$ of crack initiation (the so-called hot spot) on the notch surface (see Fig. 2 where, for the sake of simplicity, a plane stress/strain situation is considered) is assumed as that point experiencing the maximum value of $\sigma_{e q, a} \quad$ (Eq. 3). Hence, considering the coordinate system $x y$, we have:

$$
\left(x_{H}, y_{H}\right)=\max _{x, y \in S_{n}}\left\{\sigma_{e q, a}(x, y)\right\}
$$

where $S_{n}$ indicates the notch surface. Note that, under any applied multiaxial loading, principal stress directions on the notch surface are fixed with respect to time and, hence, averaged principal stress directions are those depicted in Fig. 2 ( $\hat{1}$ is tangent to the notch surface and $\hat{3}$ is normal to such a surface). Consequently, the maximisation procedure (Eq. 4) indicates that the hot spot corresponds to the point (on the notch surface) where the amplitude of the maximum principal stress $\sigma_{1}$ attains the greatest value.

The orientation of the critical plane at point $\mathrm{H}$ is computed by considering the off angle $\delta$ (Fig. 2). Then, it is assumed that such an orientation does not change up to point $P$ which is at a distance $L / 2$ ( $L$ is given in Eq. 1) from the notch surface (Fig. 2). In line with the point method of Taylor [7], the equivalent normal stress $\sigma_{e q, a}$ (Eq. 3) is calculated from the stress tensor (obtained from linear-elastic analysis) at point $P$, bearing in mind that the orientation of the critical plane (where to calculate the normal $N_{\max }$ and shear stress $C_{a}$ components) depends on the averaged principal stress directions at point $\mathrm{H}$ and the off angle 
$\delta$. The limiting cases for the critical plane orientation are as follows: if $\tau_{a f} / \sigma_{a f}=1$ the critical plane HP is normal to the notch surface, while if $\tau_{\text {af }} / \sigma_{\text {af }}=1 / \sqrt{3}$ the critical plane $\mathrm{HP}$ is $45^{\circ}$ off the normal to the notch surface.

Note that if two principal stress components (e.g. $\sigma_{2}=\sigma_{3}=0$ ) are null at point $\mathrm{H}$ (plane stress condition), two principal directions are indeterminate (e.g. in the plane normal to the principal direction of $\sigma_{1}$ ) and, hence, also the critical plane orientation is indeterminate. In such a case, it is assumed that the principal direction of $\sigma_{3}$ belongs to the stress plane (xy plane in Fig. 2), so that the critical plane is that of maximum stress gradient.

From the stress tensor at point $P$, the averaged direction $\hat{1}$ of the maximum principal stress can be calculated. It can be conjectured, although this is beyond the scope of the present paper, that the crack plane during Stage 2 propagation (final fatigue fracture plane) is normal to the direction $\hat{1}$ at point $P$ (Fig. 2).

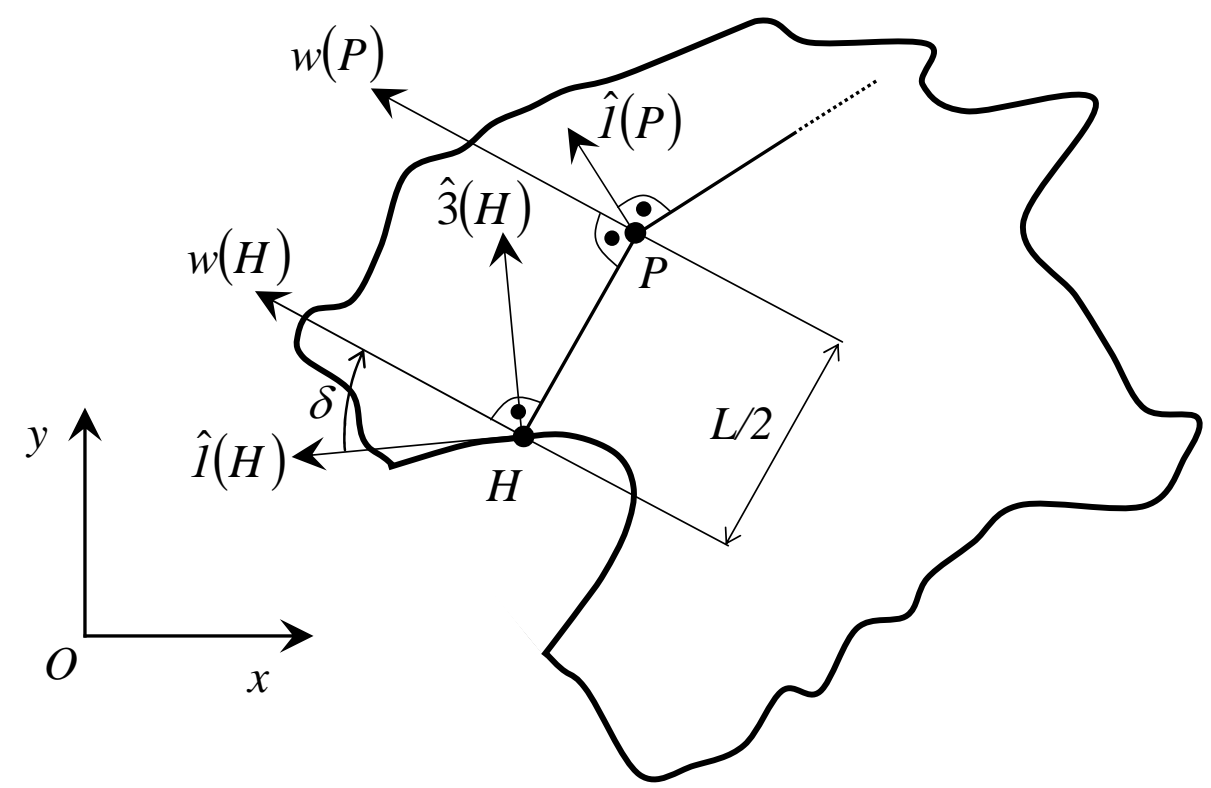

Figure 2. Critical plane orientation in the vicinity of a general traction-free notch surface (plane stress/strain conditions are assumed).

\section{Comparison with experimental results for circular notches}

Some experimental tests by Endo [24,25] are considered for comparison. The experiments concern round bars with artificially drilled surface holes (the hole diameter $D$ ranges from 40 to $500 \mu \mathrm{m}$ ). The bars are subjected to fully reversed bending, torsion and combined in-phase bending and torsion. The material being analysed is $0.37 \% \mathrm{C}$ annealed steel (with Vickers hardness equal to $\left.160, \sigma_{a f}=233 \mathrm{MPa}, \tau_{a f}=145 \mathrm{MPa}, \Delta K_{t h}=7.6 \mathrm{MPa} \sqrt{\mathrm{m}, L}=340 \mu \mathrm{m}\right)$. The threshold range of the stress intensity factor is estimated from the values reported in Ref. [26]. From Eq. 2, the off angle $\delta$ is equal to $41^{\circ}$.

The applied far-field stress amplitudes $\sigma_{x, a}$ (due to bending) and $\tau_{x y, a}$ (due to torsion) are shown in Fig. 3 , where the xy plane is tangent to the bar surface in the hole center. Stress states in the vicinity of the hole are here determined according to the Kirsch solution for plane stress state [27]. The different values of the biaxiality ratio $\lambda=\tau_{x y, a} / \sigma_{x, a}$ being considered in the experimental tests are: 0 (pure bending), $0.5,1.0,2.0, \infty$ (pure torsion). 


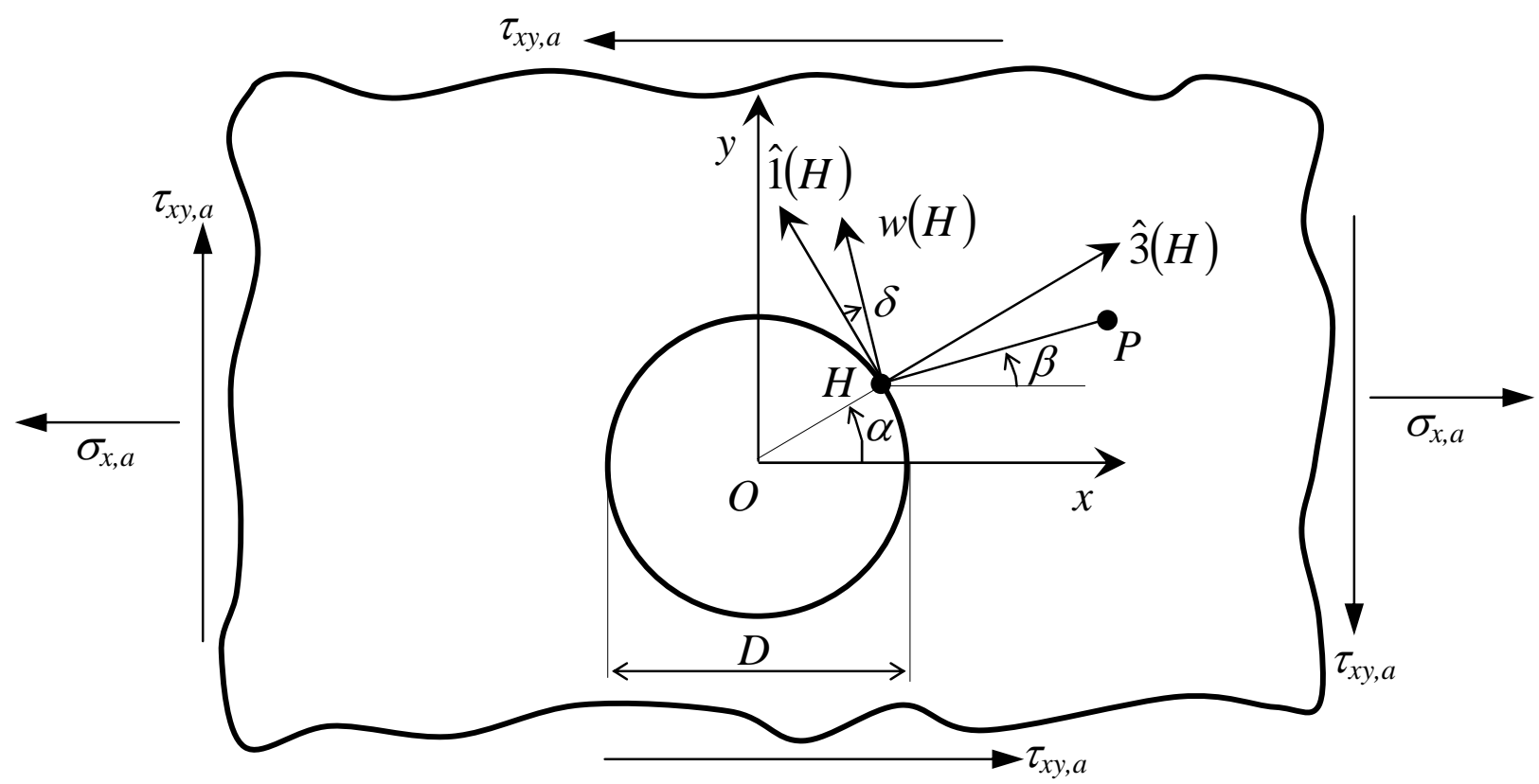

Figure 3. Critical plane orientation in the vicinity of a circular hole under in-phase axial and shear stresses (plane stress conditions are assumed).

Table 1. Position of the hot spot on the hole surface and direction of the critical plane.

\begin{tabular}{c|cc}
\hline$\lambda$ & $|\alpha|$ (deg) & $|\beta|(\mathrm{deg})$ \\
\hline 0.0 & 90 & $90-\delta$ \\
0.5 & 70 & $70-\delta$ \\
1.0 & 60 & $60-\delta$ \\
2.0 & 50 & $50-\delta$ \\
$\infty$ & 45 & $45-\delta$ \\
\hline
\end{tabular}

In Table 1, the hot spot position (see angle $\alpha$ in Fig.3) as a function of the different values of the biaxiality ratio is reported along with the critical plane orientation (see angle $\beta$ in Fig.3).

Figure 4 illustrates the experimental results by Endo [24,25] for pure bending, torsion and combined bending and torsion, and theoretical results determined through the C-S criterion. The comparison is quite satisfactory with an error within about $25 \%$. It is worth noticing that, in a recent paper by Taylor [28], similar experimental data were compared with results deduced by combining the line method of Taylor [8] with the Susmel-Lazzarin criterion [29]. In particular, different paths emanating from the hole were considered to average stresses according to the line method. It is interesting to note that Taylor [28] concluded that $45^{\circ}$ paths gave the best comparisons, which is in line with the results of the present investigation.

\section{Conclusions}

The present study represents a first attempt to predict fatigue limit conditions for multiaxially-loaded notched components by combining a critical plane-based criterion recently proposed by the authors (C-S criterion) with a critical-distance method. In particular, following the philosophy of the point method, fatigue limit conditions occur in a notched component submitted to farfield multiaxial loading when the amplitude of the equivalent normal stress, defined according to the C-S criterion, attains the normal stress fatigue limit for plain components in a point at a distance from the hot spot on the notch surface which is half the ElHaddad 'intrinsic' crack length. Such a distance is measured along a direction which depends on the material fatigue limit ratio. The comparison with some experimental results concerning circular notches casts a promising light on the present proposal. 


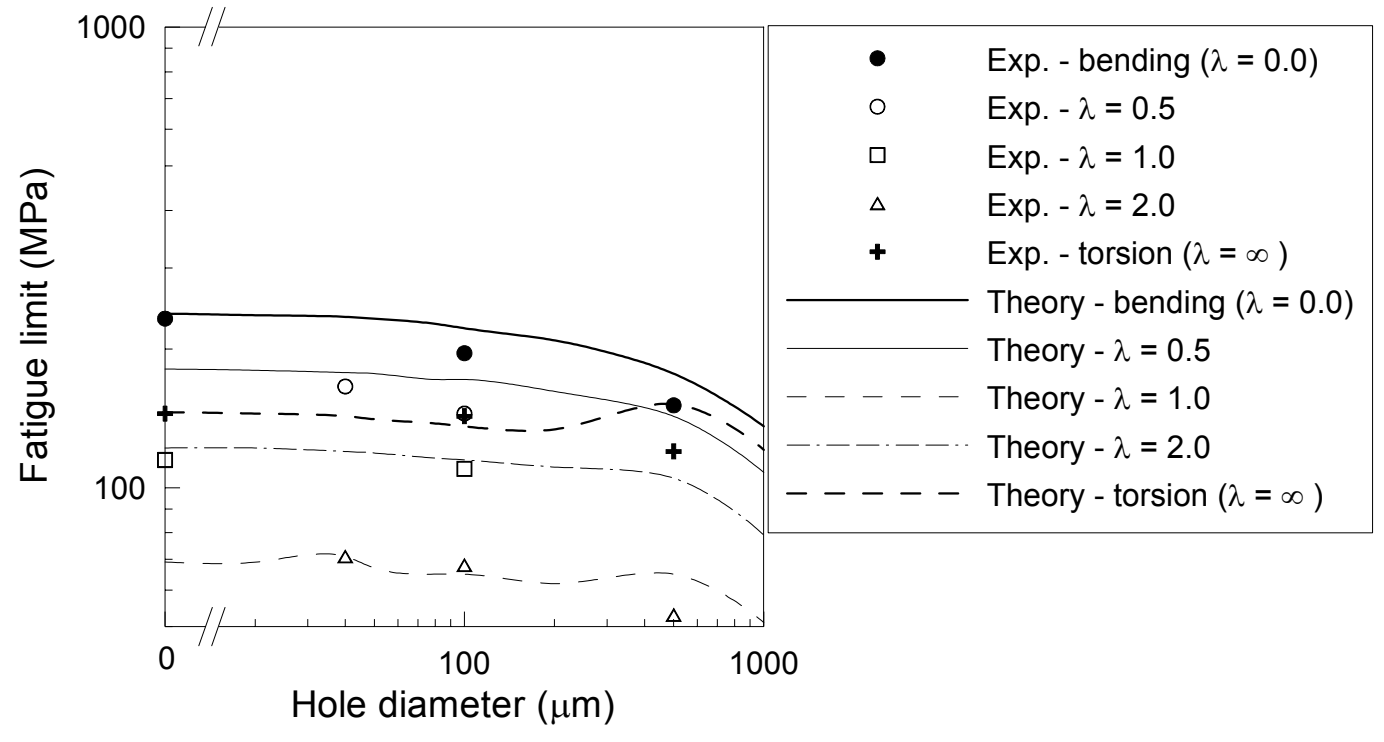

Figure 4. Experimental results [24,25] and theoretical predictions of fatigue limit as a function of the hole diameter for bending (in terms of applied normal stress amplitude), torsion (in terms of applied shear stress amplitude) and combined bending and torsion (in terms of applied normal stress amplitude).

\section{Acknowledgements}

The authors gratefully acknowledge the research support for this work provided by the Italian Ministry for University and Technological and Scientific Research (MIUR).

\section{References}

1. Neuber, H., Theory of Notch Stresses: Principles for Exact Stress Calculation, Edwards (1946).

2. Peterson, R.E., "Notch sensitivity", In: Metal Fatigue (Eds Sines, G. and Waisman J.L.), McGraw-Hill, pp. $293-306$ (1959)

3. Flavenot, J.-F. and Skalli, N.A., "A critical depth criterion for the evaluation of long-life fatigue strength under multiaxial loading and a stress gradient", In: Biaxial and Multiaxial Fatigue ECF 3 (Eds Brown, M.W. and Miller K.J.), Mechanical Engineering Publications, pp. 355-365 (1989).

4. Seweryn, A. and Mroz, Z., "On the criterion of damage evolution for variable multiaxial stress states", Int. J. Solids Structs, 35, 1589-1616 (1998).

5. Papadopoluos, I.V. and Panoskaltsis, V.P., "Invariant formulation of a gradient dependent multiaxial high-cycle fatigue criterion", Engng Fract. Mechs, 55, 513-528 (1996).

6. Tanaka, K., "Engineering formulae for fatigue strength reduction due to crack-like notches", Int. J. Fract. 22, R39-R45 (1983).

7. Taylor, D., "Geometrical effects in fatigue: a unifying theoretical model", Int. J. Fatigue, 21, 413-420 (1999).

8. ElHaddad, M.H., Dowling N.F., Topper, T.H. and Smith, K.N., "J integral applications for short fatigue cracks at notches", Int. J. Fract., 16, 15-24 (1980).

9. Taylor, D. and Wang, G., "The validation of some methods of notch fatigue analysis", Fatigue Fract. Engng Mater. Struct., 23, 387-394 (2000).

10. Smith, R.A. and Miller, K.J., "Prediction of fatigue regimes in notched components", Int. J. Mech. Sci., 20, 201-206 (1978).

11. Taylor, D., "A mechanistic approach to critical-distance methods in notch fatigue", Fatigue Fract. Engng Mater. Struct., 24, 215-224 (2001).

12. Susmel, L. and Taylor, D., "Two methods for predicting the multiaxial fatigue limits of sharp notches", Fatigue Fract. Engng Mater. Struct., 26, 821-833 (2003).

13. Naik, R.A., Lanning, D.B., Nicholas, T. and Kallmeyer, A.R., "A critical plane gradient approach for the prediction of notched HCF life", Int. J. Fatigue 27, 481-492 (2005).

14. Carpinteri, A., Brighenti, R., Macha, E. and Spagnoli A., "Expected principal stress directions for multiaxial random loading-part I: theoretical aspects of the weight function method", Int. J. Fatigue, 21, 83-88 (1999). 
15. Carpinteri, A., Brighenti, R., Macha, E. and Spagnoli A., "Expected principal stress directions for multiaxial random loading - Part II: Numerical simulation and experimental assessment through the weight function method", Int. J. Fatigue, 21, 8996 (1999).

16. Carpinteri, A., Brighenti, R. and Spagnoli A., "A fracture plane approach in multiaxial high-cycle fatigue of metals", Fatigue Fract. Engng Mater. Struct., 23, 355-364 (2000).

17. Carpinteri, A. and Spagnoli, A., "Multiaxial high-cycle fatigue criterion for hard metals", Int. J. Fatigue, 23, 135-145 (2001).

18. Spagnoli, A. "A new high-cycle fatigue criterion applied to out-of-phase biaxial stress state", Int. J. Mechanical Sci., 43, 2581-2595 (2001).

19. Carpinteri, A., Spagnoli, A. and Vantadori, S., "A multiaxial fatigue criterion for random loading", Fatigue Fract. Engng Mater. Struct., 26, 515-522 (2003).

20. Brown, M.W. and Miller, K.J., "A theory for fatigue failure under multiaxial stress-strain condition", Proc. Institute Mech. Engineers, 187, 745-755 (1973).

21. Socie, D.F., "Multiaxial fatigue damage models", J. Engng Mater. Techn., 109, 293-298 (1987).

22. Papadopoulos, I.V., "Critical plane approaches in high-cycle fatigue: on the definition of the amplitude and mean value of the shear stress acting on the critical plane", Fract. Engng Mater. Struct., 21, 269-285 (1998).

23. Gough, H.J., Pollard, H.V. and Clenshaw, W.J., "Some experiments on the resistance of metals to fatigue under combined stresses", Aeronautical Res. Council Reports, R and M 2522, HMSO (1951).

24. Endo, M., "Effects of small defects on the fatigue strength of steel and ductile iron under combined axial/torsional loading", In: Small Fatigue Cracks: Mechanics, Mechanisms and Applications (Eds Ravichandran, K.S., Ritchie, R.O. and Murakami, Y.), Elsevier, pp. 375-387 (1999).

25. Endo, M., "The multiaxial fatigue strength of specimens containing small defects", In: Biaxial/Multiaxial Fatigue and Fracture ESIS Publication 31 (Eds Carpinteri, A., de Freitas, M. and Spagnoli, A.), Elsevier, pp. 243-264 (2003).

26. Murakami, Y., Metal Fatigue: Effects of Small Defects and Nonmetallic Inclusions, Elsevier (2002).

27. Kirsh, G., "Die teorie der elastizität und die bedürfnisse der festigkeitslehre“, V.D.I., 42, 797-807 (1898).

28. Taylor, D., "Notch size effects in multiaxial fatigue", In: Progettazione a fatica in presenza di multiassialità tensionali (Eds Susmel, L. and Tovo, R.), SG Editoriali, pp. 3-13 (2005).

29. Susmel, L. and Lazzarin, P., "A bi-parametric Wohler curve for high cycle multiaxial fatigue assessment", Fatigue Fract. Engng Mater. Struct., 25, 63-78 (2002). 